\title{
Adaptive Neural Network Control with Backstepping for Surface Ships with Input Dead-Zone
}

\author{
Guoqing Xia, Xingchao Shao, Ang Zhao, and Huiyong Wu \\ College of Automation, Harbin Engineering University, 150001 Harbin, China \\ Correspondence should be addressed to Xingchao Shao; shaoxingchao@163.com
}

Received 17 June 2013; Revised 27 August 2013; Accepted 28 August 2013

Academic Editor: Jyh-Horng Chou

Copyright (C) 2013 Guoqing Xia et al. This is an open access article distributed under the Creative Commons Attribution License, which permits unrestricted use, distribution, and reproduction in any medium, provided the original work is properly cited.

\begin{abstract}
This paper addresses the problem of adaptive neural network controller with backstepping technique for fully actuated surface vessels with input dead-zone. The combination of approximation-based adaptive technique and neural network system is used for approximating the nonlinear function of the ship plant. Through backstepping and Lyapunov theory synthesis, an indirect adaptive network controller is derived for dynamic positioning ships without dead-zone property. In order to improve the control effect, a dead-zone compensator is derived using fuzzy logic technique to handle the dead-zone nonlinearity. The main advantage of the proposed controller is that it can be designed without explicit knowledge about the ship motion model, and dead-zone nonlinearity is well compensated. A set of simulations is carried out to verify the performance of the proposed controller.
\end{abstract}

\section{Introduction}

Ship moving in the sea performs strong nonlinear and coupled property. It is difficult for researchers to obtain the ship motion model. Most modern nonlinear control theories, such as sliding mode control, backstepping technique, and feedback linearization, are mostly based on the knowledge about system models. For these control strategies, the controller cannot be derived without explicit knowledge about the system model. Meanwhile, dead-zone characteristic is quite common in actuators, that is, rudders or propellers. But few works consider the influences on control performance brought by the dead-zone characteristic.

Fuzzy logic system and neural network are commonly used for approximating the unknown terms or uncertainties in the systems [1-6]. An adaptive fuzzy decentralized output-feedback control problem was discussed for a class of nonlinear large-scale systems, and fuzzy logic systems were employed to approximate the unknown nonlinear functions in the control design in [1]. Zhu and Li proposed a stable decentralized adaptive fuzzy sliding mode control scheme for reconfigurable modular manipulators to satisfy the concept of modular software in [2], and a first-order TakagiSugeno fuzzy logic system was introduced to approximate the unknown dynamics of subsystem by using adaptive algorithm. The fuzzy basis function was used to approximate an unknown nonlinear function according to some adaptive laws in [3], and then the state observer was designed for estimating the states of the plant, upon which an adaptive fuzzy sliding mode controller was investigated. In [4], a radial basis function (RBF) network were employed to estimate and compensate the uncertainties of ship dynamics and disturbances in controller design. In [5], an adaptive neural network control scheme for robot manipulators with actuator nonlinearities was presented, and the RBF network was introduced to emulate the unknown parameters. An adaptive RBF neural network controller was adopted to learn the unknown upper bound of model uncertainties and external disturbances in [6]. The approach taken in this paper tries to overcome the necessity for ship's mathematical models by using an adaptive RBF network control algorithm to estimate the unknown nonlinear functions. Meanwhile, adaptive RBF network can improve the robustness of the control systems.

In real applications, the dead-zone characteristic is quite common in actuators. Sometimes the dead-zone nonlinearity affects the control system performance. Generally, the dead-zone parameters are unknown and time-variant, which cause challenging problem for the control system. Dead-zone commonly affects all practical systems, such as 
mechanics and electronics, so the study on dead-zone has drawn much interest in the control community for a long time [7-12]. To solve the problem brought by the deadzone, adaptive dead-zone inverses were proposed in [79]. Reference [7] built a continuous dead-zone inverse for linear system with unmeasurable dead-zone outputs, while asymptotical adaptive cancellation of an unknown deadzone was achieved analytically under the condition that the output of a dead-zone was measurable in [8]. For a general nonlinear actuator dead-zone of unknown width, [9] presented a compensation scheme using neural network. In [11], adaptive control with adaptive dead-zone inverse has been introduced by giving a matching condition to the reference model. By utilizing the integral-type Lyapunov function and introducing an adaptive compensation for the upper bound of the optimal approximation error and the dead-zone disturbance, a robust adaptive neural controller for a single input single output nonlinear system was derived in [12]. By utilizing a description of a dead-zone feature, an adaptive law was used to estimate the properties of the deadzone model intuitively and mathematically, without constructing a dead-zone inverse in [13]. Departing from existing approximate adaptive dead-zone compensations, [14] used indirect parameter estimation algorithms along with online condition monitoring to obtain an accurate estimation of the unknown dead-zone when certain relaxed persistentexcitation conditions are satisfied-a theoretical result that cannot be achieved with the existing methods.

This paper proposes the work on adaptive neural network control with backstepping technique for fully actuated surface vessels with constraint in the actuator. The main contributions of this paper are as follows.

(i) To the best of our knowledge, it is the first time in the literature that both input saturation and deadzone are considered during controller design for fully actuated surface ships motion control problems.

(ii) For handling the limit of propellers, the auxiliary design system is introduced to analyze the input saturation, and the effect of dead-zone nonlinearity is compensated by using fuzzy logic system (FLS).

(iii) The adaptive method and radial basis function neural network (RBFNN) system are combined to estimate the unknown nonlinear functions of the ship plant.

(iv) Under the assumptions of the inexistence of the input saturation and the dead-zone characteristic in actuator, an adaptive RBFNN controller using backstepping technique for fully actuated surface ships is derived without knowing the ship motion model.

The main advantage of the proposed method is that the controller can be designed without explicit knowledge about the ship motion model, and control input saturation and dead-zone nonlinearity are well compensated.

The rest of this paper is organized as follows. In Section 2, a model of fully actuated surface vessels with dead-zone is established. Section 3 contains the design of adaptive RBFNN controller with backstepping, the auxiliary design system is introduced to handle the input saturation, and an FLS is

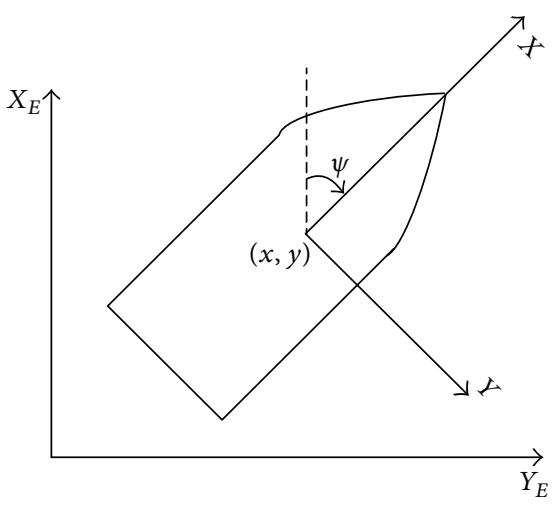

Figure 1: Definition of the earth-fixed $X_{E} Y_{E} Z_{E}$ and the body-fixed $X Y Z$ reference frames.

utilized to approximate the nonlinearity of the dead-zone in actuators. Then a set of simulations is taken in Section 4 to verify the control effect of the proposed method. Finally, conclusions are made in Section 5.

\section{Model of Surface Vessels}

2.1. Ship Motion Model for Surface Vessels. For the horizontal motion of a fully actuated surface vessel, the kinematics and dynamics models can be described by (1), for more details see [15].

$$
\begin{gathered}
\dot{\boldsymbol{\eta}}=\mathrm{J}(\boldsymbol{\eta}) \boldsymbol{v} \\
\mathrm{M} \dot{v}+\mathrm{C}(\boldsymbol{v}) \boldsymbol{v}+\mathrm{D}(\boldsymbol{v}) \boldsymbol{v}=\boldsymbol{\tau}+\mathbf{R}^{\mathrm{T}}(\boldsymbol{\eta}) \mathbf{b}
\end{gathered}
$$

where $\boldsymbol{\eta}=[x, y, \psi]^{\mathrm{T}}, \boldsymbol{\nu}=[u, v, r]^{\mathrm{T}}$, and $x, y$ denote the coordinates of the vessel in the earth-fixed frame $X_{E} Y_{E} Z_{E}$ (see Figure 1), and $\psi$ is the heading angle of the ship. $u, v$, and $r$ express the velocities in surge, sway, and yaw, respectively in the body-fixed reference frame XYZ, $\tau=\left[\tau_{x}, \tau_{y}, \tau_{\psi}\right]^{\mathrm{T}}$ represents the control inputs, that is, forces and moments produced by the propellers, $\mathbf{M}$ stands for the mass and inertia matrix, $\mathbf{C}(\boldsymbol{v})$ denotes the Coriolis-centripetal matrix, $\mathbf{D}(\boldsymbol{v})$ is the damping matrix, and $\mathbf{b}$ is a bias term representing slowly varying environmental forces and moments caused by the wind, second-order waves, and currents. $\mathbf{J}(\boldsymbol{\eta})$ is a state dependent transformation matrix which can be written as

$$
\mathbf{J}(\boldsymbol{\eta})=\mathbf{J}(\psi)=\left[\begin{array}{ccc}
\cos \psi & -\sin \psi & 0 \\
\sin \psi & \cos \psi & 0 \\
0 & 0 & 1
\end{array}\right]
$$

Note that the matrix $\mathbf{J}(\boldsymbol{\eta})$ is nonsingular for all $\boldsymbol{\eta} \in \mathbb{R}^{3}$ and $\mathbf{J}^{-1}(\boldsymbol{\eta})=\mathbf{J}^{\mathrm{T}}(\boldsymbol{\eta})$; for example, $\mathbf{J}(\boldsymbol{\eta}) \mathbf{J}^{\mathrm{T}}(\boldsymbol{\eta})=\mathbf{I}$.

The system model can be rewritten as [16]

$$
\mathbf{M}_{\eta}(\boldsymbol{\eta}) \ddot{\boldsymbol{\eta}}+\mathrm{C}_{\eta}(\boldsymbol{\nu}, \boldsymbol{\eta}) \dot{\boldsymbol{\eta}}+\mathrm{D}_{\eta}(\boldsymbol{\nu}, \boldsymbol{\eta}) \dot{\boldsymbol{\eta}}=\mathrm{J}^{-\mathrm{T}}(\boldsymbol{\eta}) \boldsymbol{\tau}+\mathbf{b},
$$


where

$$
\begin{gathered}
\mathbf{M}_{\eta}(\boldsymbol{\eta})=\mathbf{J}^{-\mathrm{T}}(\boldsymbol{\eta}) \mathbf{M J}^{-1}(\boldsymbol{\eta}), \\
\mathbf{C}_{\eta}(\boldsymbol{\nu}, \boldsymbol{\eta})=\mathbf{J}^{-\mathrm{T}}(\boldsymbol{\eta})\left[\mathbf{C}(\boldsymbol{\nu})-\mathbf{M J}^{-1}(\boldsymbol{\eta}) \dot{\mathbf{J}}(\boldsymbol{\eta})\right] \mathbf{J}^{-1}(\boldsymbol{\eta}), \\
\mathbf{D}_{\eta}(\boldsymbol{\nu}, \boldsymbol{\eta})=\mathbf{J}^{-\mathrm{T}}(\boldsymbol{\eta}) \mathbf{D}(\boldsymbol{\nu}) \mathbf{J}^{-1}(\boldsymbol{\eta}) .
\end{gathered}
$$

Considering the presence of input saturation constraints on $\boldsymbol{\tau}$, we have $\tau_{i \min } \leq \tau_{i} \leq \tau_{i \max }(i=x, y, \psi)$, where $\tau_{i \text { min }}$ and $\tau_{i \max }$ are the known lower limit and upper limit of input saturation constraints. Thus, the control input is defined as

$$
\tau_{i}= \begin{cases}\tau_{i \max }, & \tau_{0 i}>\tau_{i \max }, \\ \tau_{0 i}, & \tau_{i \min } \leq \tau_{0 i} \leq \tau_{i \max } \\ \tau_{i \min }, & \tau_{0 i}<\tau_{i \min },\end{cases}
$$

where $\tau_{0 i}$ is the $i$ th control input of the designed control law $\tau_{0}$.

Assume that the system parameters are unknown and bounded and the following properties are satisfied.

(i) $\mathbf{M}_{\eta}(\boldsymbol{\eta})$ is a positive definite matrix, and it is bounded, which means that there exists a constant $\sigma_{0}>0$, such that $\mathbf{0}<\mathbf{M}_{\eta}(\boldsymbol{\eta}) \leq \sigma_{0} \mathbf{I}$, where $\mathbf{I}$ is a 3rd-order identity matrix.

(ii) The matrix $\mathbf{M}_{\eta}(\boldsymbol{\eta})$ and $\mathbf{C}_{\eta}(\boldsymbol{\nu}, \boldsymbol{\eta})$ have the following property: the matrix $\dot{\mathbf{M}}_{\eta}(\boldsymbol{\eta})-2 \mathbf{C}_{\eta}(\boldsymbol{\nu}, \boldsymbol{\eta})$ is skewsymmetric and for all $\mathbf{x} \in \mathbb{R}^{3}$ the following relation is satisfied:

$$
\mathbf{x}^{\mathrm{T}}\left(\dot{\mathbf{M}}_{\eta}(\boldsymbol{\eta})-2 \mathbf{C}_{\eta}(\boldsymbol{\nu}, \boldsymbol{\eta})\right) \mathbf{x}=0, \quad \forall \boldsymbol{\nu}, \boldsymbol{\eta} \in \mathbb{R}^{3} .
$$

2.2. Model for Controller Design. In order to discuss conveniently and simplify the controller design, state transformation is introduced here to obtain a new form of the system model.

Let $\mathbf{x}_{1}=\boldsymbol{\eta}$ and $\mathbf{x}_{2}=\dot{\boldsymbol{\eta}}$ then the system model (1) can be written as;

$$
\begin{gathered}
\dot{\mathbf{x}}_{1}=\mathbf{x}_{2}, \\
\dot{\mathbf{x}}_{2}=\mathbf{M}_{\eta}^{-1}(\boldsymbol{\eta}) \mathbf{u}-\mathbf{M}_{\eta}^{-1}(\boldsymbol{\eta}) \mathbf{C}_{\eta}(\boldsymbol{\nu}, \boldsymbol{\eta}) \mathbf{x}_{2} \\
-\mathbf{M}_{\eta}^{-1}(\boldsymbol{\eta}) \mathbf{D}_{\eta}(\boldsymbol{\nu}, \boldsymbol{\eta}) \mathbf{x}_{2}+\mathbf{M}_{\eta}^{-1}(\boldsymbol{\eta}) \mathbf{b},
\end{gathered}
$$

where $\mathbf{u}=\mathrm{J}^{-\mathrm{T}}(\boldsymbol{\eta}) \boldsymbol{\tau}=\mathbf{J}(\boldsymbol{\eta}) \boldsymbol{\tau}$.

\section{Control Strategy}

An adaptive fuzzy logic control using backstepping technique is designed for fully actuated surface vessels with dead-zone in actuator. To compensate the dead-zone nonlinearity, a fuzzy logic system is introduced.

\subsection{Backstepping Design without Dead-Zone}

Step 1. Define the desired position and heading vector as $\boldsymbol{\eta}_{d}$, and a perfect guidance system is introduced to generate the tracking target $\boldsymbol{\eta}_{r}$; and its first- and second-order derivation $\dot{\boldsymbol{\eta}}_{r}$ and $\ddot{\boldsymbol{\eta}}_{r}$ are also generated. Then the control objective is to design a controller to make the ship track the target $\boldsymbol{\eta}_{\boldsymbol{r}}$. Define the tracking error as

$$
\mathbf{z}_{1}=\boldsymbol{\eta}-\boldsymbol{\eta}_{r} .
$$

Taking the time derivation of (9) yields

$$
\dot{\mathbf{z}}_{1}=\dot{\boldsymbol{\eta}}-\dot{\boldsymbol{\eta}}_{r}=\dot{\mathbf{x}}_{1}-\dot{\boldsymbol{\eta}}_{r}=\mathbf{x}_{2}-\dot{\boldsymbol{\eta}}_{r} .
$$

Choose $\mathbf{x}_{2}$ as virtual control and defined as

$$
\xi_{1} \triangleq \mathbf{x}_{2}=\mathbf{z}_{2}+\boldsymbol{\alpha}_{1},
$$

where $\mathbf{z}_{2}$ is the second error vector, and $\boldsymbol{\alpha}_{1}$ is the stable function which is defined later.

Substitute (11) into (10), we obtain

$$
\dot{\mathbf{z}}_{1}=\mathbf{x}_{2}-\dot{\boldsymbol{\eta}}_{r}=\mathbf{z}_{2}+\boldsymbol{\alpha}_{1}-\dot{\boldsymbol{\eta}}_{r} .
$$

Choose the stable function $\boldsymbol{\alpha}_{1}$ as

$$
\boldsymbol{\alpha}_{1}=-\lambda_{1} \mathbf{z}_{1}+\dot{\boldsymbol{\eta}}_{r},
$$

where $\lambda_{1}$ is a positive definite diagonal matrix.

The Lyapunov function is chosen for the $\mathbf{z}_{1}$-subsystem as

$$
V_{1}=\frac{1}{2} \mathbf{z}_{1}^{\mathrm{T}} \mathbf{z}_{1}
$$

Then the time derivation of (14) is

$$
\begin{aligned}
\dot{V}_{1} & =\mathbf{z}_{1}^{\mathrm{T}} \dot{\mathbf{z}}_{1}=\mathbf{z}_{1}^{\mathrm{T}}\left(\mathbf{z}_{2}+\boldsymbol{\alpha}_{1}-\dot{\boldsymbol{\eta}}_{r}\right) \\
& =-\mathbf{z}_{1}^{\mathrm{T}} \boldsymbol{\lambda}_{1} \mathbf{z}_{1}+\mathbf{z}_{1}^{\mathrm{T}} \mathbf{z}_{2} .
\end{aligned}
$$

If $\mathbf{z}_{2}=0$, then $\dot{V}_{1} \leq 0$ which implies that the $\mathbf{z}_{1}$-subsystem is stable.

Step 2. From (11) we can get

$$
\mathbf{z}_{2}=\mathbf{x}_{2}-\boldsymbol{\alpha}_{1} .
$$

Differencing (16) with respect to time yields

$$
\dot{\mathbf{z}}_{2}=\dot{\mathbf{x}}_{2}-\dot{\boldsymbol{\alpha}}_{1} .
$$

Substitute (8) into (17), then (17) becomes

$$
\dot{\mathbf{z}}_{2}=\mathbf{M}_{\eta}^{-1} \mathbf{u}-\mathbf{M}_{\eta}^{-1} \mathbf{C}_{\eta} \mathbf{x}_{2}-\mathbf{M}_{\eta}^{-1} \mathbf{D}_{\eta} \mathbf{x}_{2}+\mathbf{M}_{\eta}^{-1} \mathbf{b}-\dot{\boldsymbol{\alpha}}_{1} .
$$

Note that, we omit the variables of coefficient matrices for denoting conveniently.

For convenience of constraint effect analysis of the input saturation, the following auxiliary design system is given by

$$
\dot{\zeta}= \begin{cases}-\mathbf{K}_{1} \zeta-\frac{\left|\mathbf{z}_{2}^{\mathrm{T}} \Delta \mathbf{u}\right|+0.5 \Delta \mathbf{u}^{\mathrm{T}} \Delta \mathbf{u}}{\|\zeta\|^{2}} \zeta+\Delta \mathbf{u}, & \|\zeta\| \geq \mu, \\ \mathbf{0}, & \|\zeta\|<\mu,\end{cases}
$$

where $\Delta \mathbf{u}=\mathbf{u}-\mathbf{u}_{0}=\mathbf{J}(\boldsymbol{\eta})\left(\boldsymbol{\tau}-\boldsymbol{\tau}_{0}\right), \mathbf{K}_{1}=\mathbf{K}_{1}^{\mathrm{T}}>0, \mu$ is a small positive design parameter, and $\zeta \in \mathbb{R}^{3}$ is the state 
of the auxiliary design system. Control command $\boldsymbol{\tau}_{0}$ will be designed later.

For $\mathbf{z}_{2}$-subsystem, the Lyapunov function is chosen as

$$
V_{2}=V_{1}+\frac{1}{2} \zeta^{\mathrm{T}} \zeta+\frac{1}{2} \mathbf{z}_{2}^{\mathrm{T}} \mathbf{M}_{\eta} \mathbf{z}_{2}
$$

Take the time derivation of (20), which yields

$$
\begin{aligned}
\dot{V}_{2}= & \dot{V}_{1}+\mathbf{z}_{2}^{\mathrm{T}} \mathbf{M}_{\eta} \dot{\mathbf{z}}_{2}+\boldsymbol{\zeta}^{\mathrm{T}} \dot{\boldsymbol{\zeta}}+\frac{1}{2} \mathbf{z}_{2}^{\mathrm{T}} \dot{\mathbf{M}}_{\eta} \mathbf{z}_{2} \\
= & -\mathbf{z}_{1}^{\mathrm{T}} \boldsymbol{\lambda}_{1} \mathbf{z}_{1}+\mathbf{z}_{1}^{\mathrm{T}} \mathbf{z}_{2}+\mathbf{z}_{2}^{\mathrm{T}} \mathbf{M}_{\eta}\left(\dot{\mathbf{x}}_{2}-\dot{\boldsymbol{\alpha}}_{1}\right)+\frac{1}{2} \mathbf{z}_{2}^{\mathrm{T}} \dot{\mathbf{M}}_{\eta} \mathbf{z}_{2}+\boldsymbol{\zeta}^{\mathrm{T}} \dot{\boldsymbol{\zeta}} \\
= & -\mathbf{z}_{1}^{\mathrm{T}} \boldsymbol{\lambda}_{1} \mathbf{z}_{1}+\mathbf{z}_{1}^{\mathrm{T}} \mathbf{z}_{2}+\mathbf{z}_{2}^{\mathrm{T}} \mathbf{M}_{\eta}\left(\mathbf{M}_{\eta}^{-1} \mathbf{u}-\mathbf{M}_{\eta}^{-1} \mathbf{C}_{\eta} \mathbf{x}_{2}\right. \\
& \left.+\frac{1}{2} \mathbf{z}_{2}^{\mathrm{T}} \dot{\mathbf{M}}_{\eta} \mathbf{z}_{2}+\boldsymbol{\zeta}^{\mathrm{T}} \dot{\boldsymbol{\zeta}} \quad \mathbf{D}_{\eta} \mathbf{x}_{2}+\mathbf{M}_{\eta}^{-1} \mathbf{b}-\dot{\boldsymbol{\alpha}}_{1}\right) \\
\leq & -\mathbf{z}_{1}^{\mathrm{T}} \boldsymbol{\lambda}_{1} \mathbf{z}_{1}+\mathbf{z}_{1}^{\mathrm{T}} \mathbf{z}_{2}+\mathbf{z}_{2}^{\mathrm{T}}\left(\mathbf{u}-\mathbf{C}_{\eta} \mathbf{x}_{2}-\mathbf{D}_{\eta} \mathbf{x}_{2}+\mathbf{b}-\mathbf{M}_{\eta} \dot{\boldsymbol{\alpha}}_{1}\right) \\
& +\frac{1}{2} \mathbf{z}_{2}^{\mathrm{T}} \dot{\mathbf{M}}_{\eta} \mathbf{z}_{2}-\boldsymbol{\zeta}^{\mathrm{T}}\left(\mathbf{K}_{1}-\frac{1}{2} \mathbf{I}_{3}\right) \boldsymbol{\zeta}-\left|\mathbf{z}_{2}^{\mathrm{T}} \boldsymbol{\Delta} \boldsymbol{\tau}\right| .
\end{aligned}
$$

According to the skew-symmetric property of the matrix $\dot{\mathbf{M}}_{\eta}(\boldsymbol{\eta})-2 \mathbf{C}_{\eta}(\boldsymbol{\nu}, \boldsymbol{\eta})$ in (6), we have $(1 / 2) \mathbf{z}_{2}^{\mathrm{T}} \dot{\mathbf{M}}_{\eta} \mathbf{z}_{2}=\mathbf{z}_{2}^{\mathrm{T}} \mathbf{C}_{\eta}(\boldsymbol{\nu}, \boldsymbol{\eta}) \mathbf{z}_{2}$. Substituting it into (21) yields

$$
\begin{aligned}
\dot{V}_{2} \leq & -\mathbf{z}_{1}^{\mathrm{T}} \boldsymbol{\lambda}_{1} \mathbf{z}_{1}+\mathbf{z}_{1}^{\mathrm{T}} \mathbf{z}_{2}+\mathbf{z}_{2}^{\mathrm{T}} \\
& \times\left(\mathbf{u}+\mathbf{C}_{\eta} \mathbf{z}_{2}-\mathbf{C}_{\eta} \mathbf{x}_{2}-\mathbf{D}_{\eta} \mathbf{x}_{2}+\mathbf{b}-\mathbf{M}_{\eta} \dot{\boldsymbol{\alpha}}_{1}\right) \\
& -\boldsymbol{\zeta}^{\mathrm{T}}\left(\mathbf{K}_{1}-\frac{1}{2} \mathbf{I}_{3}\right) \zeta-\left|\mathbf{z}_{2}^{\mathrm{T}} \Delta \boldsymbol{\tau}\right| .
\end{aligned}
$$

Invoking (11) and (22) becomes

$$
\begin{aligned}
\dot{V}_{2} \leq & -\mathbf{z}_{1}^{\mathrm{T}} \boldsymbol{\lambda}_{1} \mathbf{z}_{1}+\mathbf{z}_{1}^{\mathrm{T}} \mathbf{z}_{2}+\mathbf{z}_{2}^{\mathrm{T}}\left(\mathbf{u}-\mathbf{C}_{\eta} \boldsymbol{\alpha}_{1}-\mathbf{D}_{\eta} \mathbf{x}_{2}-\mathbf{M}_{\eta} \dot{\boldsymbol{\alpha}}_{1}\right) \\
& +\mathbf{z}_{2}^{\mathrm{T}} \mathbf{b}-\boldsymbol{\zeta}^{\mathrm{T}}\left(\mathbf{K}_{1}-\frac{1}{2} \mathbf{I}_{3}\right) \zeta-\left|\mathbf{z}_{2}^{\mathrm{T}} \Delta \boldsymbol{\tau}\right| .
\end{aligned}
$$

Let $\mathbf{f}=-\mathbf{C}_{\eta} \boldsymbol{\alpha}_{1}-\mathbf{D}_{\eta} \mathbf{x}_{2}-\mathbf{M}_{\eta} \dot{\boldsymbol{\alpha}}_{1}$, and (23) can be written as

$$
\begin{aligned}
\dot{V}_{2} \leq & -\mathbf{z}_{1}^{\mathrm{T}} \boldsymbol{\lambda}_{1} \mathbf{z}_{1}+\mathbf{z}_{1}^{\mathrm{T}} \mathbf{z}_{2}+\mathbf{z}_{2}^{\mathrm{T}}(\mathbf{u}+\mathbf{f}+\mathbf{b}) \\
& -\boldsymbol{\zeta}^{\mathrm{T}}\left(\mathbf{K}_{1}-\frac{1}{2} \mathbf{I}_{3}\right) \zeta-\left|\mathbf{z}_{2}^{\mathrm{T}} \Delta \boldsymbol{\tau}\right| .
\end{aligned}
$$

Then the ideal control law can be chosen as

$$
\mathbf{u}_{0}^{*}=-\lambda_{2}\left(\mathbf{z}_{2}-\zeta\right)-\mathbf{z}_{1}-\widehat{\mathbf{f}}+\mathbf{v},
$$

where $\boldsymbol{\lambda}_{2}$ is a positive definite diagonal matrix, $\widehat{\mathbf{f}}$ is a nonlinear function designed later, and $\mathbf{v}$ is a robust term for disturbance and estimated error.

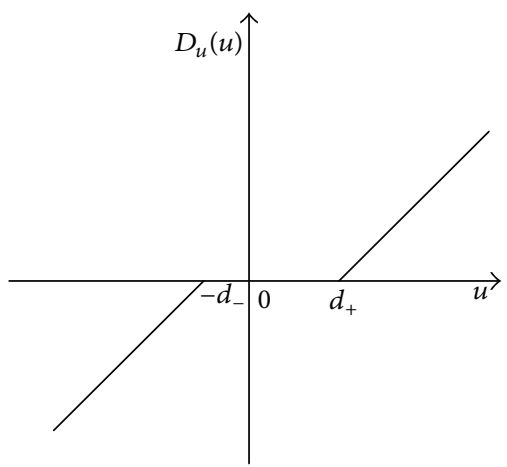

FIGURE 2: Unsymmetrical dead-zone nonlinearity.

3.2. Dead-Zone Compensator Design. The unsymmetrical dead-zone nonlinearity is shown as Figure 2, and it can be described as

$$
\tau=D_{u}(u)= \begin{cases}u+d_{-}, & u<-d_{-}, \\ 0, & -d_{-} \leq u<d_{+}, \\ u-d_{+}, & u \geq d_{+}\end{cases}
$$

where $u$ is the control input before the dead-zone, $\tau$ is the output of dead-zone model, and $d_{-}, d_{+}$are unknown positive constant. Then the dead-zone model can be denoted as

$$
\begin{gathered}
\tau=u-\operatorname{sat}_{d}(u), \\
\text { sat }_{d}(u)= \begin{cases}-d_{-}, & u<-d_{-}, \\
u, & -d_{-} \leq u<d_{+}, \\
d_{+}, & u \geq d_{+} .\end{cases}
\end{gathered}
$$

For multiple input multiple output system such as dynamics positioning ships, control input of the ith control loop is

$$
\tau_{i}=D\left(u_{i}\right)=u_{i}-\operatorname{sat}_{d_{i}}(u),
$$

where $d_{i}=\left[d_{i+}, d_{i-}\right]^{\mathrm{T}}$, and let $\mathbf{D}=\operatorname{diag}\left\{d_{1}, \ldots, d_{n}\right\}$, it becomes

$$
\boldsymbol{\tau}=\mathbf{u}-\operatorname{sat}_{D}(\mathbf{u}) .
$$

According to the characteristic of dead-zone, the rules for compensation are designed as

$$
\begin{aligned}
& \text { if }\left(w_{i} \text { is positive }\right) \text {, then }\left(u_{i}=w_{i}+\widehat{d}_{i+}\right), \\
& \text { if }\left(w_{i} \text { is negative }\right), \text { then }\left(u_{i}=w_{i}+\widehat{d}_{i-}\right),
\end{aligned}
$$

and $\widehat{\mathbf{d}}_{i}=\left[\widehat{d}_{i+}, \widehat{d}_{i_{-}}\right]^{\mathrm{T}}$ is estimate of dead-zone $\mathbf{d}_{i}=\left[d_{i+}, d_{i_{-}}\right]^{\mathrm{T}}$.

After compensating the dead-zone, the control input $u_{i}$ is

$$
u_{i}=w_{i}+w_{F_{i}}
$$

where $w_{F_{i}}$ is a compensation term which is determined by

$$
\begin{gathered}
\text { if }\left(w_{i} \in X_{+}\left(w_{i}\right)\right) \text {, then }\left(w_{F_{i}}=\widehat{d}_{i+}\right) \\
\text { if }\left(w_{i} \in X_{-}\left(w_{i}\right)\right) \text {, then }\left(w_{F_{i}}=-\widehat{d}_{i-}\right),
\end{gathered}
$$


where $X_{+}(\cdot), X_{-}(\cdot)$ are the membership degree of $w_{i}$ and $\widehat{\mathbf{d}}_{i}=$ $\left[\widehat{d}_{i+}, \widehat{d}_{i-}\right]^{\mathrm{T}}$. Let $\mathbf{w}_{F}=\left[w_{F_{1}}, \ldots, w_{F_{n}}\right]^{\mathrm{T}}$; then the control action after compensation is

$$
\mathbf{u}=\mathbf{w}+\mathbf{w}_{F}=\mathbf{w}+\widehat{\mathbf{D}}^{\mathrm{T}} X(\mathbf{w}),
$$

where $\widehat{\mathbf{D}}=\operatorname{diag}\left\{\widehat{\mathbf{d}}_{1}, \ldots, \widehat{\mathbf{d}}_{n}\right\}$ and $X(\mathbf{w})$ is described as

$$
X(\mathbf{w})=\left[X_{+}\left(w_{1}\right),-X_{-}\left(w_{1}\right), \ldots, X_{+}\left(w_{n}\right),-X_{-}\left(w_{n}\right)\right]^{\mathrm{T}} .
$$

Then membership of $w_{i}$ is designed as

$$
\begin{aligned}
& X_{+}\left(w_{i}\right)= \begin{cases}0, & w_{i}<0, \\
1, & w_{i} \geq 0,\end{cases} \\
& X_{-}\left(w_{i}\right)= \begin{cases}1, & w_{i}<0, \\
0, & w_{i} \geq 0 .\end{cases}
\end{aligned}
$$

According to Theorem 1 in [17], the control input after the dead-zone is

$$
\boldsymbol{\tau}=\mathbf{w}-\widetilde{\mathbf{D}}^{\mathrm{T}} X(\mathbf{w})+\widetilde{\mathbf{D}}^{\mathrm{T}} \boldsymbol{\delta}
$$

where $\widetilde{\mathbf{D}}=\mathbf{D}-\widehat{\mathbf{D}}=\operatorname{diag}\left\{\widetilde{d}_{1}, \ldots, \widetilde{d}_{1}\right\}$ and $\|\boldsymbol{\delta}\| \leq \sqrt{n}$.

With the control input chosen as $(25)$ and $\mathbf{w}=J^{-1}(\eta) \mathbf{u}_{0}^{*}$, the time derivation of $V_{2}$ becomes

$$
\begin{aligned}
\dot{V}_{2} \leq & -\mathbf{z}_{1}^{\mathrm{T}} \boldsymbol{\lambda}_{1} \mathbf{z}_{1}+\mathbf{z}_{1}^{\mathrm{T}} \mathbf{z}_{2}+\mathbf{z}_{2}^{\mathrm{T}}\left[-\boldsymbol{\lambda}_{2}\left(\mathbf{z}_{2}-\zeta\right)-\mathbf{z}_{1}-\widehat{\mathbf{f}}+\mathbf{f}+\mathbf{b}\right. \\
& \left.+\mathbf{v}-\mathbf{J}^{-1} \widetilde{\mathbf{D}}^{\mathrm{T}} X(\mathbf{w})+\mathbf{J}^{-1} \widetilde{\mathbf{D}}^{\mathrm{T}} \boldsymbol{\delta}\right] \\
& -\boldsymbol{\zeta}^{\mathrm{T}}\left(\mathbf{K}_{1}-\frac{1}{2} \mathbf{I}_{3}\right) \zeta-\left|\mathbf{z}_{1}^{\mathrm{T}} \Delta \tau\right| \\
\leq & -\mathbf{z}_{1}^{\mathrm{T}} \boldsymbol{\lambda}_{1} \mathbf{z}_{1}-\mathbf{z}_{2}^{\mathrm{T}} \boldsymbol{\lambda}_{2}\left(\mathbf{z}_{2}-\boldsymbol{\zeta}\right) \\
& +\mathbf{z}_{2}^{\mathrm{T}}\left(\mathbf{f}-\widehat{\mathbf{f}}+\mathbf{v}+\mathbf{b}-\mathbf{J}^{-1} \widetilde{\mathbf{D}}^{\mathrm{T}} X(\mathbf{w})+\mathbf{J}^{-1} \widetilde{\mathbf{D}}^{\mathrm{T}} \boldsymbol{\delta}\right) \\
& -\boldsymbol{\zeta}^{\mathrm{T}}\left(\mathbf{K}_{1}-\frac{1}{2} \mathbf{I}_{3}\right) \boldsymbol{\zeta} .
\end{aligned}
$$

We can choose the robust term and adaptive law of deadzone width as

$$
\begin{gathered}
\mathbf{v}(t)=-\mathbf{b}_{M} \operatorname{sgn}\left(\mathbf{z}_{2}\right), \\
\dot{\widehat{\mathbf{D}}}=-\boldsymbol{\Gamma} X(\mathbf{w}) \mathbf{z}_{2}^{\mathrm{T}} \mathbf{J}^{-1}-\frac{1}{2} \chi \widehat{\mathbf{D}},
\end{gathered}
$$

where $\boldsymbol{\chi}>0, \Gamma>0$ are diagonal matrices and $0 \leq\|\mathbf{b}\| \leq \mathbf{b}_{M}$.

3.3. Adaptive RBFNN Controller Design. From the description of $\mathbf{f}$ mentioned above, we can see that it contains the knowledge about the system model. In order to design a control law without explicit knowledge about the ship motion model, an adaptive RBFNN is introduced to approximate the nonlinear function $\mathbf{f}$. And here $\widehat{\mathbf{f}}$ is the adaptive neural network system used to approximate $\mathbf{f}$.

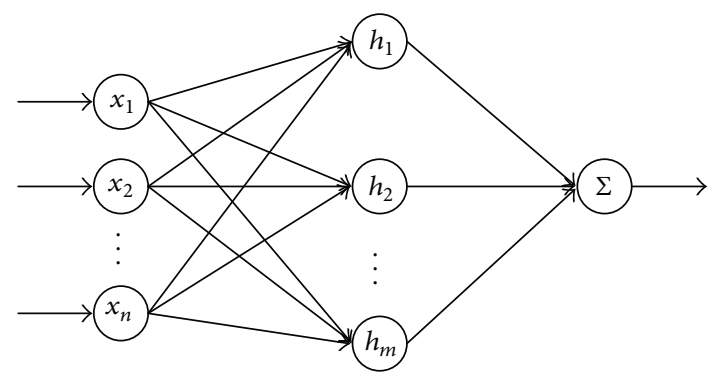

FIgURE 3: The structure of RBFNN.

The structure of RBFNN is shown in Figure 3. RBFNN is a forward network with three layers: input layer, implicit layer, and output layer. The mapping from input to output is nonlinear, while it is linear from implicit layer to output. RBFNN can approximate nonlinear function locally, so its learning rate is fast and the local minimization problem can be avoided.

Assume there are $n$ inputs and $m$ implicits nodes in the RBF network. Let $\mathbf{X}=\left[x_{1}, x_{2}, \ldots, x_{n}\right]^{\mathrm{T}}$ be the input vector; then denote the radial basis vector as $\mathbf{H}=\left[h_{1}, h_{2}, \ldots, h_{m}\right]^{\mathrm{T}}$, where $h_{i}$ is Gaussian function defined as

$$
h_{i}=\exp \left(-\frac{\left\|\mathbf{X}-\mathbf{c}_{i}\right\|^{2}}{2 b_{i}^{2}}\right), \quad i=1,2, \ldots, m,
$$

where $\mathbf{c}_{i}=\left[c_{i 1}, c_{i 2}, \ldots, c_{i n}\right]^{\mathrm{T}}$ is the central vector of the $i$ th node and $b_{i}>0$ is the basis width parameter of the $i$ th node.

Define the weight vector of the RBF neural network as $\mathbf{W}=\left[w_{1}, w_{2}, \ldots, w_{m}\right]^{\mathrm{T}}$; then the output of the RBFNN can be expressed as follows:

$$
y=\mathbf{H}^{\mathrm{T}} \mathbf{W} .
$$

In order to approximate the nonlinear function $\mathbf{f}$ which is relative to the ship modeled parameters and the system states, the elements of $\mathbf{f}$ are, respectively, estimated by corresponding RBFNN as follows:

$$
\widehat{f}_{k}(\mathbf{x})=\mathbf{H}_{k}(\mathbf{x})^{\mathrm{T}} \mathbf{W}_{k}, \quad(k=1,2,3) .
$$

Then define the Gaussian basis function vector $\varphi$ as

$$
\widehat{\mathbf{f}}(\mathbf{x}, \boldsymbol{\theta})=\left[\begin{array}{l}
f_{1} \\
f_{2} \\
f_{3}
\end{array}\right]=\left[\begin{array}{ccc}
\mathbf{H}_{1}^{\mathrm{T}} & \mathbf{0} & \mathbf{0} \\
\mathbf{0} & \mathbf{H}_{2}^{\mathrm{T}} & \mathbf{0} \\
\mathbf{0} & \mathbf{0} & \mathbf{H}_{3}^{\mathrm{T}}
\end{array}\right]\left[\begin{array}{l}
\mathbf{W}_{1} \\
\mathbf{W}_{2} \\
\mathbf{W}_{3}
\end{array}\right]=\boldsymbol{\xi}(\mathbf{x})^{\mathrm{T}} \boldsymbol{\theta},
$$

where $\boldsymbol{\xi}^{\mathrm{T}}(\mathbf{x})=\operatorname{diag}\left\{\mathbf{H}_{1}^{\mathrm{T}}, \mathbf{H}_{2}^{\mathrm{T}}, \mathbf{H}_{3}^{\mathrm{T}}\right\}$ and $\boldsymbol{\theta}=\left[\mathbf{W}_{1}^{\mathrm{T}}, \mathbf{W}_{2}^{\mathrm{T}}, \mathbf{W}_{3}^{\mathrm{T}}\right]^{\mathrm{T}}$.

Assumption 1. The output of $\operatorname{RBFNN} \widehat{\mathbf{f}}(\mathbf{x}, \boldsymbol{\theta})$ is continuous.

Assumption 2. There exist an ideal approximation $\widehat{\mathbf{f}}\left(\mathbf{x}, \boldsymbol{\theta}^{*}\right)$, such that for any small positive constant $\varepsilon_{0}$

$$
\max \left\|\mathbf{f}(\mathbf{x})-\widehat{\mathbf{f}}\left(\mathbf{x}, \boldsymbol{\theta}^{*}\right)\right\| \leq \varepsilon_{0}
$$

where $\boldsymbol{\theta}^{*}$ is the optimal weight vector of the RBFNN for approximating $\mathbf{f}(\mathbf{x})$. 
Denote $\mathbf{e}_{a}$ as the approximate error of the ideal RBFNN:

$$
\mathbf{e}_{a}=\mathbf{f}(\mathbf{x})-\widehat{\mathbf{f}}\left(\mathbf{x}, \boldsymbol{\theta}^{*}\right) .
$$

According to the approximate capability of RBFNN, it is easy to obtain that $\mathbf{e}_{a}$ is bounded. Denote the bound as

$$
\mathbf{e}_{a 0}=\sup \left\|\mathbf{f}(\mathbf{x})-\widehat{\mathbf{f}}\left(\mathbf{x}, \boldsymbol{\theta}^{*}\right)\right\|
$$

where $\widehat{\mathbf{f}}\left(\mathbf{x}, \boldsymbol{\theta}^{*}\right)=\boldsymbol{\xi}(\mathbf{x})^{\mathrm{T}} \boldsymbol{\theta}^{*}$.

$\mathbf{f}(\mathbf{x})$ is bounded, so $\boldsymbol{\theta}^{*}$ is bounded and $\left\|\boldsymbol{\theta}^{*}\right\|_{F} \leq \boldsymbol{\theta}_{\text {max }}$. Let $\widetilde{\boldsymbol{\theta}}=\boldsymbol{\theta}^{*}-\boldsymbol{\theta}$, and the adaptive law is chosen as

$$
\dot{\boldsymbol{\theta}}_{i}=r_{i}\left(z_{2 i} \boldsymbol{\xi}_{i}^{\mathrm{T}}(\mathbf{x})\right)^{\mathrm{T}}-2 \boldsymbol{k}_{i} \boldsymbol{\theta}_{i}, \quad(i=1,2,3),
$$

where $r_{i}>0$ and $k_{i}>0$ are designed constant and $z_{2 i}$ is the $i$ th element of $\mathbf{z}_{2}$.

Let $\boldsymbol{\gamma} \in \mathfrak{R}^{3 m \times 3 m}, \boldsymbol{\kappa} \in \mathfrak{R}^{3 m \times 3 m}$, and define matrices as

$$
\begin{aligned}
& \boldsymbol{\gamma}=\operatorname{diag}\left\{r_{1} \mathbf{I}_{m}, r_{2} \mathbf{I}_{m}, r_{3} \mathbf{I}_{m}\right\}, \\
& \boldsymbol{\kappa}=\operatorname{diag}\left\{k_{1} \mathbf{I}_{m}, k_{2} \mathbf{I}_{m}, k_{3} \mathbf{I}_{m}\right\},
\end{aligned}
$$

where $\mathbf{I}_{n}$ is a $n$th order identity matrix. Then the adaptive law (47) becomes

$$
\dot{\boldsymbol{\theta}}=\boldsymbol{\gamma}\left(\mathbf{z}_{2}^{\mathrm{T}} \boldsymbol{\xi}^{\mathrm{T}}(\mathbf{x})\right)^{\mathrm{T}}-2 \boldsymbol{\kappa} \boldsymbol{\theta} .
$$

3.4. Stability Analysis. Now we can choose the total Lyapunov function as

$$
V=V_{2}+\frac{1}{2} \widetilde{\boldsymbol{\theta}}^{\mathrm{T}} \boldsymbol{\gamma}^{-1} \widetilde{\boldsymbol{\theta}}+\frac{1}{2} \operatorname{tr}\left(\widetilde{\mathbf{D}}^{\mathrm{T}} \boldsymbol{\Gamma}^{-1} \widetilde{\mathbf{D}}\right)
$$

Differentiate (50) with respect to time as

$$
\begin{aligned}
\dot{V} \leq & \dot{V}_{2}+\widetilde{\boldsymbol{\theta}}^{\mathrm{T}} \boldsymbol{\gamma}^{-1} \dot{\tilde{\boldsymbol{\theta}}}+\operatorname{tr}\left(\widetilde{\mathbf{D}}^{\mathrm{T}} \boldsymbol{\Gamma} \dot{\widetilde{\mathbf{D}}}\right) \\
\leq & -\mathbf{z}_{1}^{\mathrm{T}} \boldsymbol{\lambda}_{1} \mathbf{z}_{1}-\mathbf{z}_{2}^{\mathrm{T}} \boldsymbol{\lambda}_{2}\left(\mathbf{z}_{2}-\boldsymbol{\zeta}\right)+\mathbf{z}_{2}^{\mathrm{T}}\left(\mathbf{f}-\boldsymbol{\xi}(\mathbf{x})^{\mathrm{T}} \boldsymbol{\theta}\right)-\widetilde{\boldsymbol{\theta}}^{\mathrm{T}} \boldsymbol{\gamma}^{-1} \dot{\boldsymbol{\theta}} \\
& -\mathbf{z}_{2}^{\mathrm{T}}\left(\mathbf{J}^{-1} \widetilde{\mathbf{D}}^{\mathrm{T}} X(\mathbf{w})-\mathbf{J}^{-1} \widetilde{\mathbf{D}}^{\mathrm{T}} \boldsymbol{\delta}\right)+\operatorname{tr}\left(\widetilde{\mathbf{D}}^{\mathrm{T}} \boldsymbol{\Gamma} \dot{\overrightarrow{\mathbf{D}}}\right) \\
& +\mathbf{z}_{2}^{\mathrm{T}}(\mathbf{b}+\mathbf{v})-\boldsymbol{\zeta}^{\mathrm{T}}\left(\mathbf{K}_{1}-\frac{1}{2} \mathbf{I}_{3}\right) \boldsymbol{\zeta} \\
\leq & -\mathbf{z}_{1}^{\mathrm{T}} \boldsymbol{\lambda}_{1} \mathbf{z}_{1}-\mathbf{z}_{2}^{\mathrm{T}} \boldsymbol{\lambda}_{2}\left(\mathbf{z}_{2}-\boldsymbol{\zeta}\right)+\mathbf{z}_{2}^{\mathrm{T}}\left(\mathbf{f}-\boldsymbol{\xi}(\mathbf{x})^{\mathrm{T}} \boldsymbol{\theta}^{*}+\mathbf{b}+\mathbf{v}\right) \\
& -\widetilde{\boldsymbol{\theta}}^{\mathrm{T}} \boldsymbol{\gamma}^{-1} \dot{\boldsymbol{\theta}}+\mathbf{z}_{2}^{\mathrm{T}} \boldsymbol{\xi}(\mathbf{x})^{\mathrm{T}}\left(\boldsymbol{\theta}^{*}-\boldsymbol{\theta}\right)-\mathbf{z}_{2}^{\mathrm{T}} \mathbf{J}^{-1} \widetilde{\mathbf{D}}^{\mathrm{T}}(X(\mathbf{w})-\boldsymbol{\delta}) \\
& +\operatorname{tr}\left(\widetilde{\mathbf{D}}^{\mathrm{T}} \boldsymbol{\Gamma} \dot{\widetilde{\mathbf{D}}}\right)-\boldsymbol{\zeta}^{\mathrm{T}}\left(\mathbf{K}_{1}-\frac{1}{2} \mathbf{I}_{3}\right) \boldsymbol{\zeta} .
\end{aligned}
$$

It is obviously that

$$
\mathbf{z}_{2}^{\mathrm{T}} \boldsymbol{\lambda}_{2} \boldsymbol{\zeta} \leq \pi \mathbf{z}_{2}^{\mathrm{T}} \mathbf{z}_{2}+\boldsymbol{\pi}^{-1} \boldsymbol{\zeta}^{\mathrm{T}} \boldsymbol{\lambda}_{2}^{\mathrm{T}} \boldsymbol{\lambda}_{2} \boldsymbol{\zeta}
$$

where $\pi \in \mathbb{R}^{3 \times 3}$ and $\pi>0$.
According to

$$
\begin{aligned}
\mathbf{z}_{2}^{\mathrm{T}}\left(\mathbf{J}^{-1} \widetilde{\mathbf{D}}^{\mathrm{T}} X(\mathbf{w})-\mathbf{J}^{-1} \widetilde{\mathbf{D}}^{\mathrm{T}} \boldsymbol{\delta}\right) & =\mathbf{z}_{2}^{\mathrm{T}} \mathbf{J}^{-1} \widetilde{\mathbf{D}}^{\mathrm{T}}(X(\mathbf{w})-\boldsymbol{\delta}) \\
& =\operatorname{tr}\left(\widetilde{\mathbf{D}}^{\mathrm{T}}(X(\mathbf{w})-\boldsymbol{\delta}) \mathbf{z}_{2}^{\mathrm{T}} \mathbf{J}^{-1}\right),
\end{aligned}
$$

we can obtain

$$
\begin{aligned}
\vartheta & \triangleq-\mathbf{z}_{2}^{\mathrm{T}}\left(\mathbf{J}^{-1} \widetilde{\mathbf{D}}^{\mathrm{T}} X(\mathbf{w})-\mathbf{J}^{-1} \widetilde{\mathbf{D}}^{\mathrm{T}} \boldsymbol{\delta}\right)+\operatorname{tr}\left(\widetilde{\mathbf{D}}^{\mathrm{T}} \boldsymbol{\Gamma}^{-1} \dot{\widetilde{\mathbf{D}}}\right) \\
& =\operatorname{tr}\left(\widetilde{\mathbf{D}}^{\mathrm{T}}(-X(\mathbf{w})+\boldsymbol{\delta}) \mathbf{z}_{2}^{\mathrm{T}} \mathbf{J}^{-1}\right)+\operatorname{tr}\left(\widetilde{\mathbf{D}}^{\mathrm{T}} \boldsymbol{\Gamma}^{-1} \dot{\widetilde{\mathbf{D}}}\right) \\
& =\operatorname{tr}\left(\widetilde{\mathbf{D}}^{\mathrm{T}}\left(-X(\mathbf{w}) \mathbf{z}_{2}^{\mathrm{T}} \mathbf{J}^{-1}+\boldsymbol{\delta} \mathbf{z}_{2}^{\mathrm{T}} \mathbf{J}^{-1}+\boldsymbol{\Gamma}^{-1} \dot{\widetilde{\mathbf{D}}}\right)\right)
\end{aligned}
$$

From $\widetilde{\mathbf{D}}=\mathbf{D}-\widehat{\mathbf{D}}$ we have $=-\dot{\widehat{\mathbf{D}}}$; then

$$
\begin{aligned}
\vartheta & =\operatorname{tr}\left(\widetilde{\mathbf{D}}^{\mathrm{T}}\left(-X(\mathbf{w}) \mathbf{z}_{2}^{\mathrm{T}} \mathbf{J}^{-1}+\boldsymbol{\delta} \mathbf{z}_{2}^{\mathrm{T}} \mathbf{J}^{-1}-\boldsymbol{\Gamma}^{-1} \dot{\hat{\mathbf{D}}}\right)\right) \\
& =\operatorname{tr}\left(\widetilde{\mathbf{D}}^{\mathrm{T}}\left(\delta \mathbf{z}_{2}^{\mathrm{T}} \mathbf{J}^{-1}+\frac{1}{2} \boldsymbol{\Gamma}^{-1}(\mathbf{D}-\widetilde{\mathbf{D}})\right)\right) \\
& \leq \sqrt{n}\left\|\mathbf{z}_{2}\right\|\|\widetilde{\mathbf{D}}\|+\frac{1}{2 g_{m}} D_{M}\|\widetilde{\mathbf{D}}\|-\frac{\chi_{m}}{2} \operatorname{tr}\left(\widetilde{\mathbf{D}}^{\mathrm{T}} \boldsymbol{\Gamma}^{-1} \widetilde{\mathbf{D}}\right),
\end{aligned}
$$

where $\chi_{m}=\sigma_{\min }(\boldsymbol{\chi}), g_{m}=\sigma_{\min }(\boldsymbol{\Gamma})$ and $D_{M}>\|\mathbf{D}\|,\|\boldsymbol{\delta}\| \leq$ $\sqrt{n}$, so $(51)$ yields

$$
\begin{aligned}
\dot{V} \leq & -\mathbf{z}_{1}^{\mathrm{T}} \boldsymbol{\lambda}_{1} \mathbf{z}_{1}-\mathbf{z}_{2}^{\mathrm{T}}\left(\boldsymbol{\lambda}_{2}-\boldsymbol{\pi}\right) \mathbf{z}_{2}+\left\|\mathbf{z}_{2}^{\mathrm{T}}\right\| \cdot\left\|\mathbf{f}-\boldsymbol{\xi}(\mathbf{x})^{\mathrm{T}} \boldsymbol{\theta}^{*}\right\| \\
& +\vartheta-\tilde{\boldsymbol{\theta}}^{\mathrm{T}} \boldsymbol{\gamma}^{-1} \dot{\boldsymbol{\theta}}+\mathbf{z}_{2}^{\mathrm{T}} \boldsymbol{\xi}(\mathbf{x})^{\mathrm{T}} \widetilde{\theta}+\mathbf{z}_{2}^{\mathrm{T}}\left(\mathbf{b}-\mathbf{b}_{M} \operatorname{sgn}\left(\mathbf{z}_{2}\right)\right) \\
& -\boldsymbol{\zeta}^{\mathrm{T}}\left(\mathbf{K}_{1}-\frac{1}{2} \mathbf{I}_{3}-\boldsymbol{\pi}^{-1} \boldsymbol{\lambda}_{2}^{\mathrm{T}} \boldsymbol{\lambda}_{2}\right) \boldsymbol{\zeta} \\
\leq & -\mathbf{z}_{1}^{\mathrm{T}} \boldsymbol{\lambda}_{1} \mathbf{z}_{1}-\mathbf{z}_{2}^{\mathrm{T}}\left(\boldsymbol{\lambda}_{2}-\boldsymbol{\pi}\right) \mathbf{z}_{2}+\frac{1}{2}\left\|\mathbf{z}_{2}^{\mathrm{T}}\right\|^{2}+\frac{1}{2} \varepsilon_{0}{ }^{2}+\vartheta \\
& +\widetilde{\boldsymbol{\theta}}^{\mathrm{T}}\left[\left(\mathbf{z}_{2}^{\mathrm{T}} \boldsymbol{\xi}^{\mathrm{T}}(\mathbf{x})^{\mathrm{T}}\right)-\boldsymbol{\gamma}^{-1} \dot{\boldsymbol{\theta}}\right] \\
& -\boldsymbol{\zeta}^{\mathrm{T}}\left(\mathbf{K}_{1}-\frac{1}{2} \mathbf{I}_{3}-\boldsymbol{\pi}^{-1} \boldsymbol{\lambda}_{2}^{\mathrm{T}} \boldsymbol{\lambda}_{2}\right) \boldsymbol{\zeta} .
\end{aligned}
$$

Substitute adaptive law (39) into (56) and we can get

$$
\begin{aligned}
\dot{V} \leq & -\mathbf{z}_{1}^{\mathrm{T}} \boldsymbol{\lambda}_{1} \mathbf{z}_{1}-\mathbf{z}_{2}^{\mathrm{T}}\left(\boldsymbol{\lambda}_{2}-\boldsymbol{\pi}\right) \mathbf{z}_{2}-\boldsymbol{\zeta}^{\mathrm{T}}\left(\mathbf{K}_{1}-\frac{1}{2} \mathbf{I}_{3}-\boldsymbol{\pi}^{-1} \boldsymbol{\lambda}_{2}^{\mathrm{T}} \boldsymbol{\lambda}_{2}\right) \boldsymbol{\zeta} \\
& +\frac{1}{2} \varepsilon^{2}-\widetilde{\boldsymbol{\theta}}^{\mathrm{T}}\left[\left(\mathbf{z}_{2}^{\mathrm{T}} \boldsymbol{\xi}^{\mathrm{T}}(\mathbf{x})^{\mathrm{T}}\right)-\boldsymbol{\gamma}^{-1}\left(\boldsymbol{\gamma}\left(\mathbf{z}_{2} \boldsymbol{\xi}^{\mathrm{T}}(\mathbf{x})\right)^{\mathrm{T}}-2 \boldsymbol{\kappa} \boldsymbol{\theta}\right)\right] \\
& +9+\frac{1}{2}\left\|\mathbf{z}_{2}^{\mathrm{T}}\right\|^{2} \\
= & -\mathbf{z}_{1}^{\mathrm{T}} \boldsymbol{\lambda}_{1} \mathbf{z}_{1}-\mathbf{z}_{2}^{\mathrm{T}}\left(\boldsymbol{\lambda}_{2}-\boldsymbol{\pi}\right) \mathbf{z}_{2}+\mathbf{z}_{2}^{\mathrm{T}} \mathbf{z}_{2}+\frac{1}{2} \varepsilon^{2} \\
& +2 \widetilde{\boldsymbol{\theta}}^{\mathrm{T}} \boldsymbol{\gamma}^{-1} \boldsymbol{\kappa} \boldsymbol{\theta}+\vartheta-\boldsymbol{\zeta}^{\mathrm{T}}\left(\mathbf{K}_{1}-\frac{1}{2} \mathbf{I}_{3}-\boldsymbol{\pi}^{-1} \boldsymbol{\lambda}_{2}^{\mathrm{T}} \boldsymbol{\lambda}_{2}\right) \boldsymbol{\zeta} \\
= & -\mathbf{z}_{1}^{\mathrm{T}} \boldsymbol{\lambda}_{1} \mathbf{z}_{1}-\mathbf{z}_{2}^{\mathrm{T}}\left(\boldsymbol{\lambda}_{2}-\frac{1}{2} \mathbf{I}_{3}\right) \mathbf{z}_{2}+\frac{1}{2} \varepsilon^{2}+2 \boldsymbol{\theta}^{* \mathrm{~T}} \boldsymbol{\gamma}^{-1} \boldsymbol{\kappa} \boldsymbol{\theta} \\
& -2 \boldsymbol{\theta}^{\mathrm{T}} \boldsymbol{\gamma}^{-1} \boldsymbol{\kappa} \boldsymbol{\theta}+\boldsymbol{\vartheta}-\boldsymbol{\zeta}^{\mathrm{T}}\left(\mathbf{K}_{1}-\frac{1}{2} \mathbf{I}_{3}-\boldsymbol{\pi}^{-1} \boldsymbol{\lambda}_{2}^{\mathrm{T}} \boldsymbol{\lambda}_{2}\right) \boldsymbol{\zeta} .
\end{aligned}
$$


If $\boldsymbol{\kappa}$ and $\gamma$ are positive definite diagonal matrices, then $\left(\boldsymbol{\theta}^{*}-\boldsymbol{\theta}\right)^{\mathrm{T}} \boldsymbol{\gamma}^{-1} \boldsymbol{\kappa}\left(\boldsymbol{\theta}^{*}-\boldsymbol{\theta}\right) \geq 0$. So the following inequality is satisfied:

$$
2 \boldsymbol{\theta}^{* \mathrm{~T}} \boldsymbol{\gamma}^{-1} \boldsymbol{\kappa} \boldsymbol{\theta}-2 \boldsymbol{\theta}^{\mathrm{T}} \boldsymbol{\gamma}^{-1} \boldsymbol{\kappa} \boldsymbol{\theta} \leq-\boldsymbol{\theta}^{\mathrm{T}} \boldsymbol{\gamma}^{-1} \boldsymbol{\kappa} \boldsymbol{\theta}+\boldsymbol{\theta}^{* \mathrm{~T}} \boldsymbol{\gamma}^{-1} \boldsymbol{\kappa} \boldsymbol{\theta}^{*} .
$$

Substituting (58) into (57) yields

$$
\begin{aligned}
\dot{V} \leq & -\mathbf{z}_{1}^{\mathrm{T}} \boldsymbol{\lambda}_{1} \mathbf{z}_{1}-\mathbf{z}_{2}^{\mathrm{T}}\left(\boldsymbol{\lambda}_{2}-\frac{1}{2} \mathbf{I}_{3}-\boldsymbol{\pi}\right) \mathbf{z}_{2}+\frac{1}{2} \varepsilon^{2}+\boldsymbol{\theta}^{* \mathrm{~T}} \boldsymbol{\gamma}^{-1} \boldsymbol{\kappa} \boldsymbol{\theta}^{*} \\
& -\boldsymbol{\theta}^{\mathrm{T}} \boldsymbol{\gamma}^{-1} \boldsymbol{\kappa} \boldsymbol{\theta}+\vartheta-\boldsymbol{\zeta}^{\mathrm{T}}\left(\mathbf{K}_{1}-\frac{1}{2} \mathbf{I}_{3}-\boldsymbol{\pi}^{-1} \boldsymbol{\lambda}_{2}^{\mathrm{T}} \boldsymbol{\lambda}_{2}\right) \boldsymbol{\zeta} \\
= & -\mathbf{z}_{1}^{\mathrm{T}} \boldsymbol{\lambda}_{1} \mathbf{z}_{1}-\mathbf{z}_{2}^{\mathrm{T}}\left(\boldsymbol{\lambda}_{2}-\frac{1}{2} \mathbf{I}_{3}-\boldsymbol{\pi}\right) \mathbf{z}_{2}-\boldsymbol{\theta}^{\mathrm{T}} \boldsymbol{\gamma}^{-1} \boldsymbol{\kappa} \boldsymbol{\theta} \\
& -\boldsymbol{\theta}^{* \mathrm{~T}} \boldsymbol{\gamma}^{-1} \boldsymbol{\kappa} \boldsymbol{\theta}^{*}+\frac{1}{2} \varepsilon^{2}+2 \boldsymbol{\theta}^{* \mathrm{~T}} \boldsymbol{\gamma}^{-1} \boldsymbol{\kappa} \boldsymbol{\theta}^{*}+\vartheta \\
& -\boldsymbol{\zeta}^{\mathrm{T}}\left(\mathbf{K}_{1}-\frac{1}{2} \mathbf{I}_{3}-\boldsymbol{\pi}^{-1} \boldsymbol{\lambda}_{2}^{\mathrm{T}} \boldsymbol{\lambda}_{2}\right) \boldsymbol{\zeta} .
\end{aligned}
$$

Due to $\left(\boldsymbol{\theta}^{*}+\boldsymbol{\theta}\right)^{\mathrm{T}} \gamma^{-1} \boldsymbol{\kappa}\left(\boldsymbol{\theta}^{*}+\boldsymbol{\theta}\right) \geq 0$, we obtain

$$
-\boldsymbol{\theta}^{* \mathrm{~T}} \boldsymbol{\gamma}^{-1} \boldsymbol{\kappa} \boldsymbol{\theta}-\boldsymbol{\theta}^{\mathrm{T}} \boldsymbol{\gamma}^{-1} \boldsymbol{\kappa} \boldsymbol{\theta}^{*} \leq \boldsymbol{\theta}^{\mathrm{T}} \boldsymbol{\gamma}^{-1} \boldsymbol{\kappa} \boldsymbol{\theta}+\boldsymbol{\theta}^{* \mathrm{~T}} \boldsymbol{\gamma}^{-1} \boldsymbol{\kappa} \boldsymbol{\theta}^{*} .
$$

Then

$$
\begin{aligned}
\widetilde{\boldsymbol{\theta}}^{\mathrm{T}} \boldsymbol{\gamma}^{-1} \boldsymbol{\kappa} \tilde{\boldsymbol{\theta}} & =\left(\boldsymbol{\theta}^{*}-\boldsymbol{\theta}\right)^{\mathrm{T}} \boldsymbol{\gamma}^{-1} \boldsymbol{\kappa}\left(\boldsymbol{\theta}^{*}-\boldsymbol{\theta}\right) \\
& =\boldsymbol{\theta}^{* \mathrm{~T}} \boldsymbol{\gamma}^{-1} \boldsymbol{\kappa} \boldsymbol{\theta}^{*}+\boldsymbol{\theta}^{\mathrm{T}} \boldsymbol{\gamma}^{-1} \boldsymbol{\kappa} \boldsymbol{\theta}-2 \boldsymbol{\theta}^{* \mathrm{~T}} \boldsymbol{\gamma}^{-1} \boldsymbol{\kappa} \boldsymbol{\theta} \\
& \leq 2 \boldsymbol{\theta}^{* \mathrm{~T}} \boldsymbol{\gamma}^{-1} \boldsymbol{\kappa} \boldsymbol{\theta}^{*}+2 \boldsymbol{\theta}^{\mathrm{T}} \boldsymbol{\gamma}^{-1} \boldsymbol{\kappa} \boldsymbol{\theta},
\end{aligned}
$$

which implies that

$$
-\boldsymbol{\theta}^{* \mathrm{~T}} \boldsymbol{\gamma}^{-1} \boldsymbol{\kappa} \boldsymbol{\theta}^{*}-\boldsymbol{\theta}^{\mathrm{T}} \boldsymbol{\gamma}^{-1} \boldsymbol{\kappa} \boldsymbol{\theta} \leq-\frac{1}{2} \widetilde{\boldsymbol{\theta}}^{\mathrm{T}} \boldsymbol{\gamma}^{-1} \boldsymbol{\kappa} \tilde{\boldsymbol{\theta}}
$$

Now (59) becomes

$$
\begin{aligned}
\dot{V} \leq & -\mathbf{z}_{1}^{\mathrm{T}} \boldsymbol{\lambda}_{1} \mathbf{z}_{1}-\mathbf{z}_{2}^{\mathrm{T}}\left(\boldsymbol{\lambda}_{2}-\frac{1}{2} \mathbf{I}_{3}-\boldsymbol{\pi}\right) \mathbf{z}_{2}-\frac{1}{2} \tilde{\boldsymbol{\theta}}^{\mathrm{T}} \boldsymbol{\gamma}^{-1} \boldsymbol{\kappa} \tilde{\boldsymbol{\theta}}+\frac{1}{2} \varepsilon^{2} \\
& +2 \boldsymbol{\theta}^{* \mathrm{~T}} \boldsymbol{\gamma}^{-1} \boldsymbol{\kappa} \boldsymbol{\theta}^{*}-\boldsymbol{\zeta}^{\mathrm{T}}\left(\mathbf{K}_{1}-\frac{1}{2} \mathbf{I}_{3}-\pi^{-1} \boldsymbol{\lambda}_{2}^{\mathrm{T}} \boldsymbol{\lambda}_{2}\right) \boldsymbol{\zeta}+\vartheta
\end{aligned}
$$

Choose appropriate $\lambda_{2}, \boldsymbol{\pi}, \mathbf{K}_{1}$ such that $\boldsymbol{\lambda}_{2}-(1 / 2) \mathbf{I}_{3}-\boldsymbol{\pi}$ and $\mathbf{K}_{1}-(1 / 2) \mathbf{I}_{3}-\pi^{-1} \lambda_{2}^{\mathrm{T}} \boldsymbol{\lambda}_{2}$ are positive definite, and $\lambda_{1 m}$, $\lambda_{2 m}, \kappa_{m}$ and $k_{m}$ are, respectively, the minimum eigenvalue of $\boldsymbol{\lambda}_{1}, \boldsymbol{\lambda}_{2}-(1 / 2) \mathbf{I}_{3}-\boldsymbol{\pi}, \boldsymbol{\kappa}$ and $\mathbf{K}_{1}-(1 / 2) \mathbf{I}_{3}-\boldsymbol{\pi}^{-1} \boldsymbol{\lambda}_{2}^{\mathrm{T}} \boldsymbol{\lambda}_{2}$. Then (63) becomes

$$
\begin{aligned}
\dot{V} \leq & -\lambda_{1 m} \mathbf{z}_{1}^{\mathrm{T}} \mathbf{z}_{1}-\lambda_{2 m} \mathbf{z}_{2}^{\mathrm{T}} \mathbf{z}_{2}-\frac{1}{2} \kappa_{m} \widetilde{\boldsymbol{\theta}}^{\mathrm{T}} \boldsymbol{\gamma}^{-1} \widetilde{\boldsymbol{\theta}} \\
& -k_{m} \boldsymbol{\zeta}^{\mathrm{T}} \boldsymbol{\zeta}+2 \boldsymbol{\theta}^{* \mathrm{~T}} \boldsymbol{\gamma}^{-1} \boldsymbol{\kappa} \boldsymbol{\theta}^{*}+\frac{1}{2} \varepsilon^{2}+\vartheta \\
= & -\lambda_{1 m} \mathbf{z}_{1}^{\mathrm{T}} \mathbf{z}_{1}-\lambda_{2 m} \mathbf{z}_{2}^{\mathrm{T}} \mathbf{M}_{\eta}^{-1} \mathbf{M}_{\eta} \mathbf{z}_{2}-k_{m} \boldsymbol{\zeta}^{\mathrm{T}} \boldsymbol{\zeta}+\frac{1}{2} \widetilde{\boldsymbol{\theta}}^{\mathrm{T}} \boldsymbol{\gamma}^{-1} \boldsymbol{\kappa} \widetilde{\boldsymbol{\theta}} \\
& +2 \boldsymbol{\theta}^{* \mathrm{~T}} \boldsymbol{\gamma}^{-1} \boldsymbol{\kappa} \boldsymbol{\theta}^{*}+\frac{1}{2} \varepsilon^{2}+\vartheta .
\end{aligned}
$$

According to $\mathbf{0}<\mathbf{M}_{\eta}(\boldsymbol{\eta}) \leq \sigma_{0} \mathbf{I}$, that is, $-\mathbf{M}_{\eta}^{-1} \leq-\left(1 / \sigma_{0}\right) ;$ then

$$
\begin{aligned}
\dot{V} \leq & -\lambda_{1 m} \mathbf{z}_{1}^{\mathrm{T}} \mathbf{z}_{1}-\frac{1}{\sigma_{0}} \lambda_{2 m} \mathbf{z}_{2}^{\mathrm{T}} \mathbf{M}_{\eta} \mathbf{z}_{2}-k_{m} \boldsymbol{\zeta}^{\mathrm{T}} \boldsymbol{\zeta}-\frac{1}{2} \kappa_{m} \widetilde{\boldsymbol{\theta}}^{\mathrm{T}} \boldsymbol{\gamma}^{-1} \widetilde{\boldsymbol{\theta}} \\
& +2 \boldsymbol{\theta}^{* \mathrm{~T}} \boldsymbol{\gamma}^{-1} \boldsymbol{\kappa} \boldsymbol{\theta}^{*}+\frac{1}{2} \varepsilon^{2}+\boldsymbol{\vartheta} .
\end{aligned}
$$

Define $c_{0}=\min \left\{2 \lambda_{1 m},\left(2 / \sigma_{0}\right) \lambda_{2 m}, \kappa_{m}, k_{m}, \chi_{m}\right\}$, then

$$
\begin{aligned}
\dot{V} \leq & -\frac{1}{2} c_{0}\left(\mathbf{z}_{1}^{\mathrm{T}} \mathbf{z}_{1}+\mathbf{z}_{2}^{\mathrm{T}} \mathbf{M}_{\eta} \mathbf{z}_{2}+\boldsymbol{\zeta}^{\mathrm{T}} \boldsymbol{\zeta}+\widetilde{\boldsymbol{\theta}}^{\mathrm{T}} \boldsymbol{\gamma}^{-1} \widetilde{\boldsymbol{\theta}}+\operatorname{tr}\left(\widetilde{\mathbf{D}}^{\mathrm{T}} \boldsymbol{\Gamma}^{-1} \widetilde{\mathbf{D}}\right)\right) \\
& +\frac{1}{2} \varepsilon^{2}+\sqrt{n}\left\|\mathbf{z}_{2}\right\|\left(\|\widetilde{\mathbf{D}}\|+\chi_{M} D_{M}\|\widetilde{\mathbf{D}}\|-\chi_{M}\|\widetilde{\mathbf{D}}\|^{2}\right) \\
& +2 \boldsymbol{\theta}^{* \mathrm{~T}} \boldsymbol{\gamma}^{-1} \boldsymbol{\kappa} \boldsymbol{\theta}^{*} \\
= & -c_{0} V+2 \boldsymbol{\theta}^{* \mathrm{~T}} \boldsymbol{\gamma}^{-1} \boldsymbol{\kappa} \boldsymbol{\theta}^{*}+\frac{1}{2} \varepsilon^{2}+\sqrt{n}\left\|\mathbf{z}_{2}\right\|\|\widetilde{\mathbf{D}}\| \\
& +\frac{1}{2 g_{m}} D_{M}\|\widetilde{\mathbf{D}}\| \\
= & -c_{0} V+c_{V M},
\end{aligned}
$$

where $c_{V M}=2 \boldsymbol{\theta}^{* \mathrm{~T}} \boldsymbol{\gamma}^{-1} \boldsymbol{\kappa} \boldsymbol{\theta}^{*}+(1 / 2) \varepsilon^{2}+\sqrt{n}\left\|\mathbf{z}_{2}\right\|\|\widetilde{\mathbf{D}}\|+$ $\left(1 / 2 g_{m}\right) D_{M}\|\widetilde{\mathbf{D}}\|>0$.

So we can obtain that $V$ and all the signals of the closeloop system are bounded [18].

\section{Case Study}

A computer simulation has been used to evaluate the performance of the adaptive neural network control with backstepping for fully actuated surface ships with dead-zone in actuator. The ship system used for simulation is described as follows:

$$
\begin{gathered}
\mathbf{M}=\left[\begin{array}{ccc}
9.1948 \times 10^{7} & 0 & 0 \\
0 & 9.1948 \times 10^{7} & 1.2607 \times 10^{9} \\
0 & 1.2607 \times 10^{9} & 1.0724 \times 10^{11}
\end{array}\right], \\
\mathbf{D}_{l}=\left[\begin{array}{ccc}
1.5073 \times 10^{6} & 0 & 0 \\
0 & 8.1687 \times 10^{6} & -1.3180 \times 10^{8} \\
0 & -1.3180 \times 10^{8} & 1.2568 \times 10^{11}
\end{array}\right], \\
\mathbf{D}_{n}(\boldsymbol{\nu})=-\operatorname{diag}\left\{X_{|u| u}|u|, Y_{|v| v}|v|, N_{|r| r}|r|\right\}
\end{gathered}
$$

where $X_{|u| u}|u|, Y_{|v| v}|v|, N_{|r| r}|r|$ are hydrodynamic coefficients, $X_{|u| u}=-2.9766 \cdot 10^{4}, Y_{|v| v}=-8.0922 \cdot 10^{4}$, and $N_{|r| r}=$ $-1.2228 \cdot 10^{12}$.

Note that the elements of matrices are the nominal values of the ship plant.

The bound of dead-zone is set as

$$
d_{-}=0.5 \times 10^{5}, \quad d_{+}=0.6 \times 10^{5} .
$$



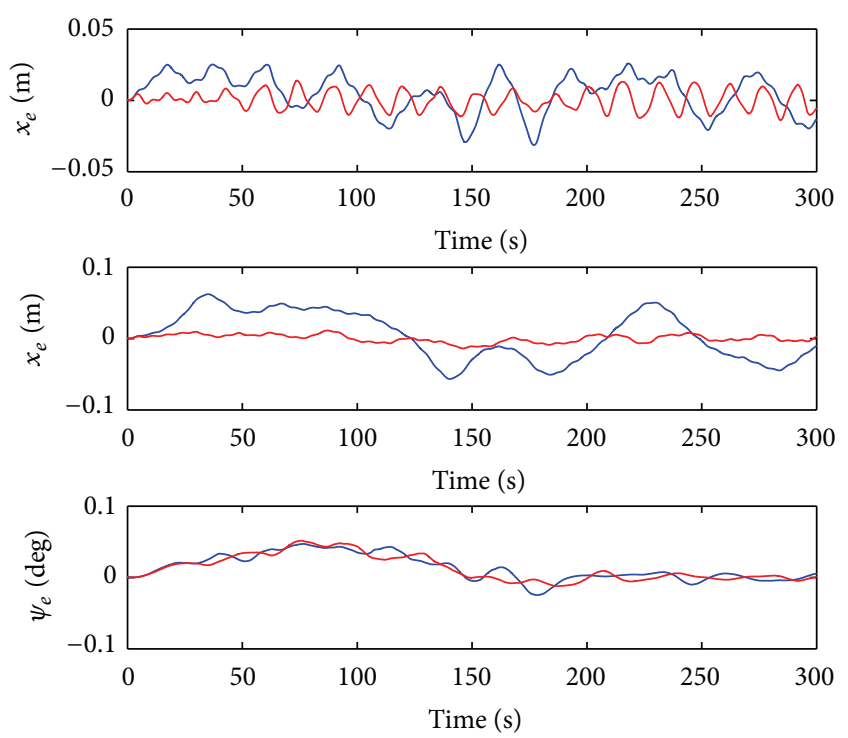

FIGURE 4: Tracking error of the position and heading.
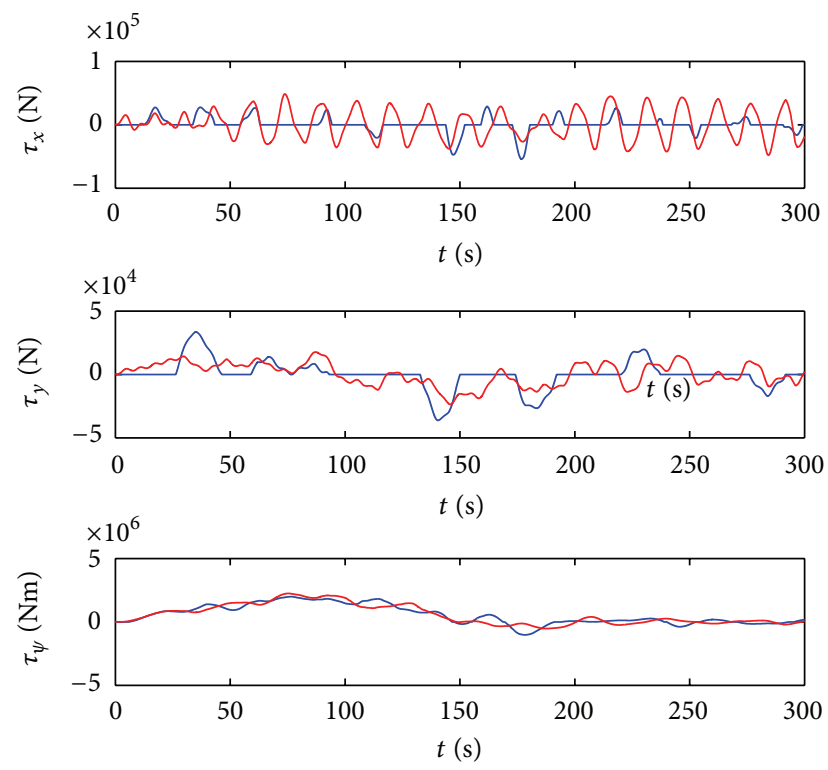

FIGURE 5: Control inputs in surge, sway, and yaw.

To verify the robustness of the controller and the control effect, the unpredictable disturbances and parameter uncertainties are introduced. The environmental disturbances acting on the ship can be treated together as

$$
\begin{aligned}
\mathbf{b}=[ & 0.25 \times 10^{5} \sin (0.1 t), 0.25 \times 10^{5} \sin (0.1 t), \\
& \left.0.25 \times 10^{6} \sin (0.1 t)\right]^{\mathrm{T}} .
\end{aligned}
$$

And parameters of the ship model vary from 0.5 to 1.5 times the size of its nominal value. The limit of control input is set as

$$
|\boldsymbol{\tau}| \leq\left[0.2 \times 10^{6}, 0.15 \times 10^{6}, 0.15 \times 10^{8}\right]^{\mathrm{T}} .
$$
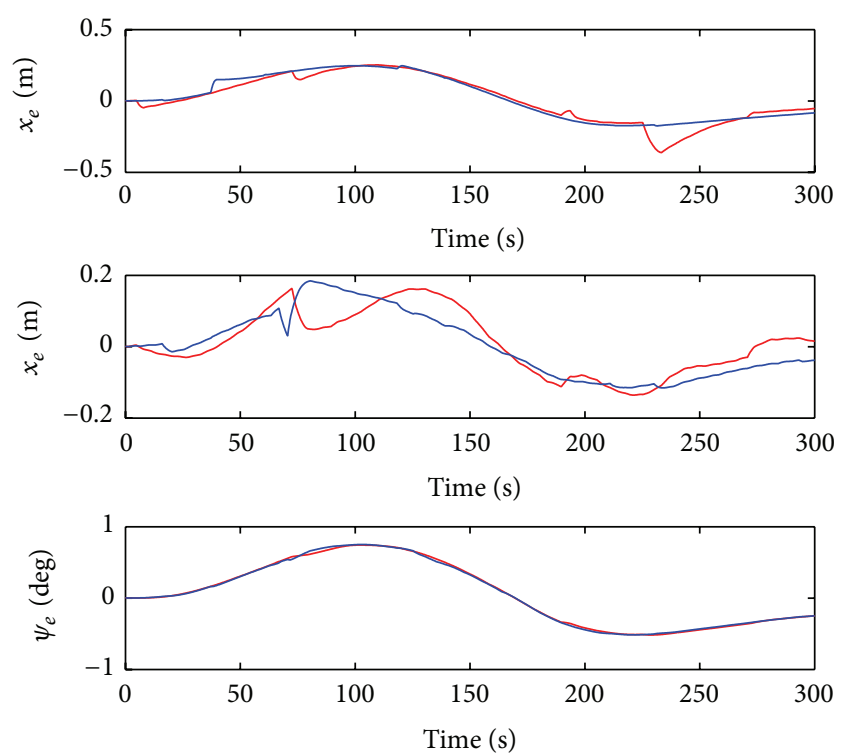

FIGURE 6: Tracking error of the position and heading with PID controller.
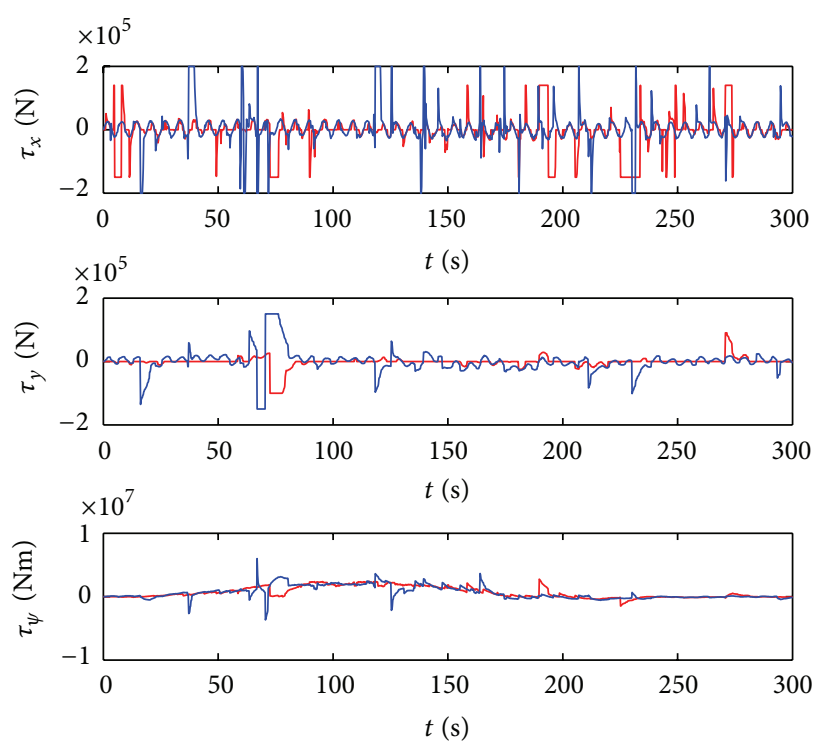

FIgURE 7: Control inputs in surge, sway, and yaw with PID controller.

The initial position and heading of the vessel is $(0 \mathrm{~m}, 0 \mathrm{~m}$, $0 \mathrm{deg})$, and the initial velocity is $(0 \mathrm{~m} / \mathrm{s}, 0 \mathrm{~m} / \mathrm{s}, 0 \mathrm{rad} / \mathrm{s})$ and the desired position is set as $(2 \mathrm{~m}, 1 \mathrm{~m}, 5 \mathrm{deg})$. A smooth reference path is generated by a guidance system. The simulation results with proposed controller are shown in Figures 4 and 5, and the ones with PID controller are listed in Figures 6 and 7. The blue lines in Figures 4 and 5 are values without using dead-zone compensator, and the red ones are the results using it. Figure 4 shows that the dead-zone compensator improves the control performance and reduces the tracking errors. Figure 5 is the control inputs for both simulations with and without dead-zone compensator. From Figure 5, the control 
input is zero when the control command is in the deadzone, so its control effect is not excellent unless the deadzone is compensated well. The blue lines in Figures 6 and 7 are results without dead-zone in actuators, and the red ones are values with dead-zone characteristic. Comparing Figures 4 and 5 to Figures 6 and 7, the method proposed in this work ameliorates the control effect, and the tracking errors are 5-10 times smaller than the results using PID controller. Meanwhile, the forces and moments acting on the ship become smaller and smoother than the ones with PID method. So the control strategy designed in this work can protect the actuator from wear and reduce fuel consumption.

\section{Conclusion}

In this paper, an adaptive neural network control using backstepping is derived for fully actuated surface ships with deadzone characteristics in actuator. A three degree of freedom model including disturbances has been established for ships. In order to overcome the difficulties brought by unknown model parameters, the adaptive RBFNN is introduced to approximate the unknown nonlinear functions needed in controller design. The dead-zone character is quite common in actuator for ships, and the existence of dead-zone affects the control effect of traditional controllers, especially when the dead-zone parameters are unknown. The fuzzy logic system is utilized here to handle this problem. Fuzzy logic technique can estimate the dead-zone parameters which are used for compensator design. It has been shown that the adaptive fuzzy control with the dead-zone compensator can drive the ship to the desired position with certain heading angles. The input saturation is overcome by adopting the auxiliary design system. Meanwhile, through the Lyapunov stable theory it is proved that the system is bounded for all states. The simulation results showed that the controller proposed in this work performs excellently for dynamic positioning ships.

\section{References}

[1] S. Tong, Y. Li, and X. Jing, "Adaptive fuzzy decentralized dynamics surface control for nonlinear large-scale systems based on high-gain observer," Information Sciences, vol. 235, pp. 287-307, 2013.

[2] M. Zhu and Y. Li, "Decentralized adaptive fuzzy sliding mode control for reconfigurable modular manipulators," International Journal of Robust and Nonlinear Control, vol. 20, no. 4, pp. 472488, 2010.

[3] C.-C. Chiang and C.-H. Wu, "Observer-based adaptive fuzzy sliding mode control of uncertain multiple-input multipleoutput nonlinear systems," in Proceedings of the IEEE International Conference on Fuzzy Systems (FUZZ-IEEE '07), pp. 1-6, London, UK, July 2007.

[4] J. L. Tao, Y. Yang, D. H. Wang, and C. Guo, "A robust adaptive neural networks controller for maritime dynamic positioning system," Neurocomputing, vol. 110, no. 1, pp. 128-136, 2013.

[5] J. Liu and Y. Lu, "Adaptive RBF neural network control of robot with actuator nonlinearities," Journal of Control Theory and Applications, vol. 8, no. 2, pp. 249-256, 2010.
[6] J. Fei and H. Ding, "Adaptive sliding mode control of dynamic system using RBF neural network," Nonlinear Dynamics, vol. 70, no. 2, pp. 1563-1573, 2012.

[7] V. K. Deolia, S. Purwar, and T. N. Sharma, "Backstepping control of discrete-time nonlinear system under unknown dead-zone constraint," in Proceedings of the International Conference on Communication Systems and Network Technologies (CSNT '11), pp. 344-349, Katra, Jammu and Kashmir, India, June 2011.

[8] H. Cho and E.-W. Bai, "Convergence results for an adaptive dead zone inverse," International Journal of Adaptive Control and Signal Processing, vol. 12, no. 5, pp. 451-466, 1998.

[9] R. R. Šelmić and F. L. Lewis, "Deadzone compensation in motion control systems using neural networks," IEEE Transactions on Automatic Control, vol. 45, no. 4, pp. 602-613, 2000.

[10] A. Taware and G. Tao, "An adaptive dead-zone inverse controller for systems with sandwiched dead-zones," International Journal of Control, vol. 76, no. 8, pp. 755-769, 2003.

[11] X.-S. Wang, H. Hong, and C.-Y. Su, "Model reference adaptive control of continuous-time systems with an unknown input dead-zone," IEEE Proceedings on Control Theory and Applications, vol. 150, no. 3, pp. 261-266, 2003.

[12] T. Zhang and S. S. Ge, "Robust adaptive neural control of SISO nonlinear systems with unknown dead-zone and completely unknown control gain," in Proceedings of the IEEE Conference on Control Applications, Computer-Aided Control Systems Design Symposium and International Symposium on Intelligent Control (ISIC '06), pp. 88-93, Munich, Germany, October 2006.

[13] C.-C. Chiang, "Adaptive fuzzy tracking control for uncertain nonlinear time-delay systems with unknown dead-zone input," Mathematical Problems in Engineering, vol. 2013, Article ID 363748, 13 pages, 2013.

[14] C. Hu, B. Yao, and Q. Wang, "Performance-oriented adaptive robust control of a class of nonlinear systems preceded by unknown dead zone with comparative experimental results," IEEE/ASME Transactions on Mechatronics, vol. 18, no. 1, pp. 178189,2013

[15] T. I. Fossen, Handbook of Marine Craft Hydrodynamics and Motion Control, John Wiley \& Sons, Chichester, UK, 2011.

[16] T. I. Fossen, Marine Control System, Guidance, Navigation and Control of Ships, Rigs and Underwater Vehicles, Trondheim, Norway, Marine Cybernetics, 2002.

[17] J. K. Liu, Design and Matlab Simulation of Robot Control System, Tsinghua University Press, Beijing, China, 2012, (Chinese).

[18] H. K. Khaill, Nonlinear System, Publishing House of Electronics Industry, Beijing, China, 2006. 


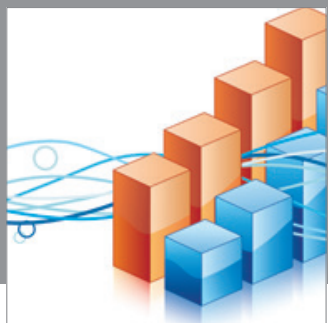

Advances in

Operations Research

mansans

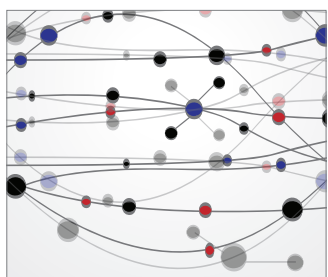

The Scientific World Journal
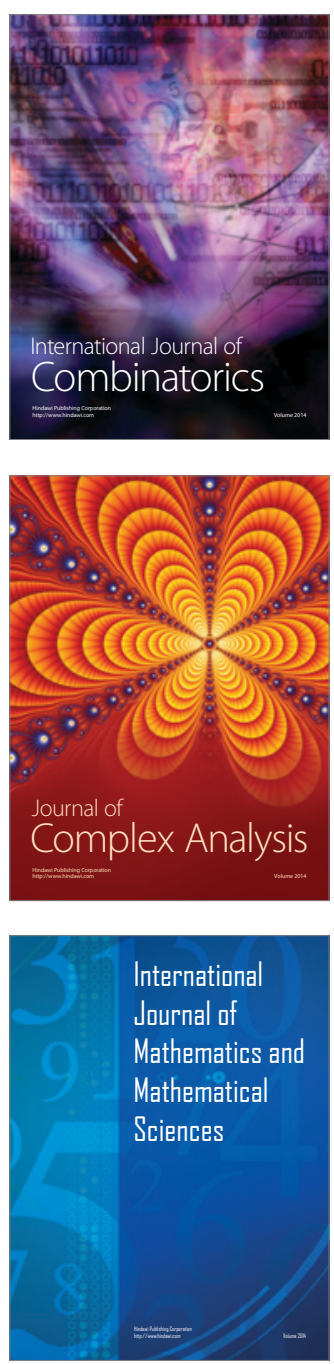
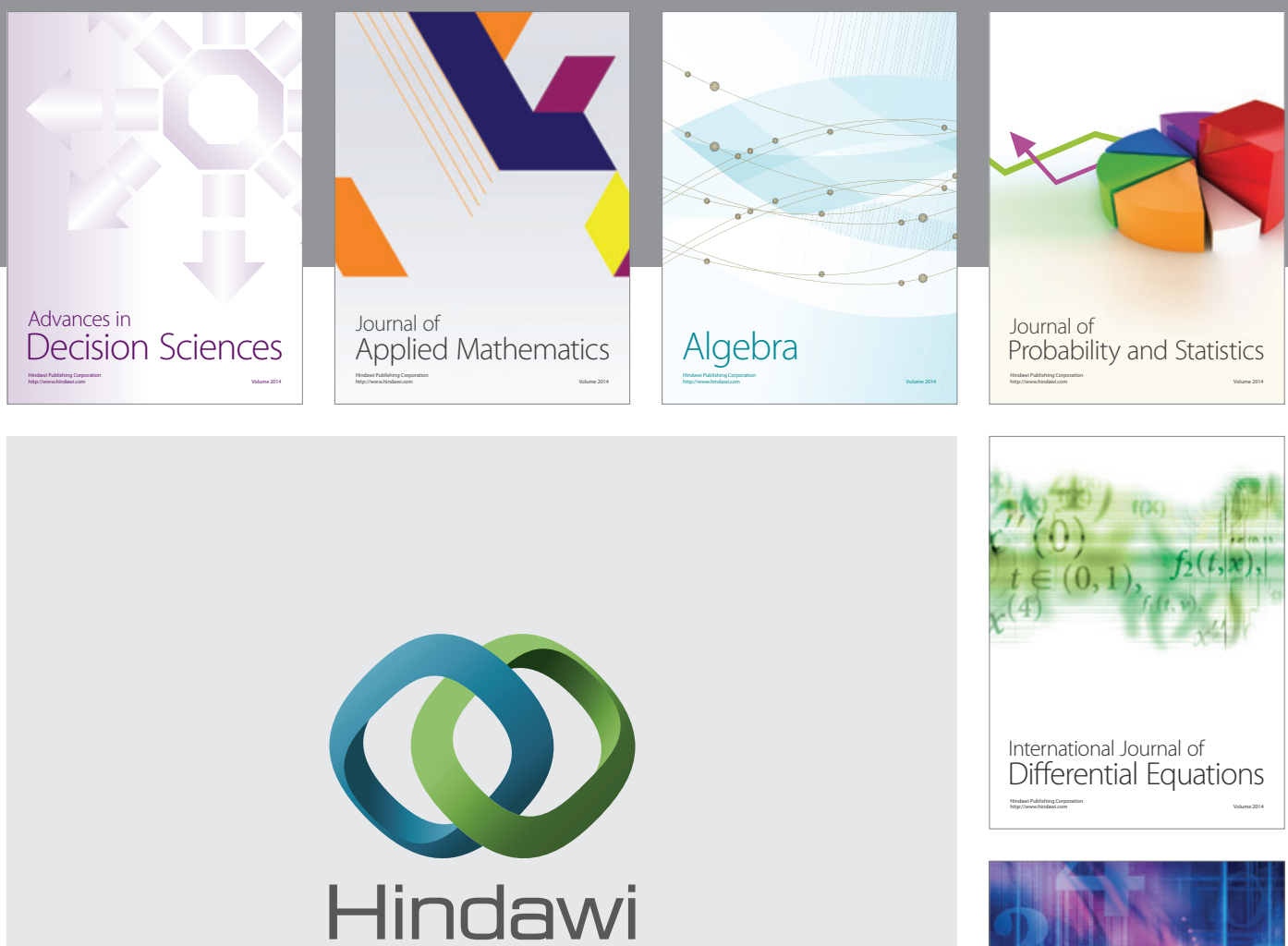

Submit your manuscripts at http://www.hindawi.com
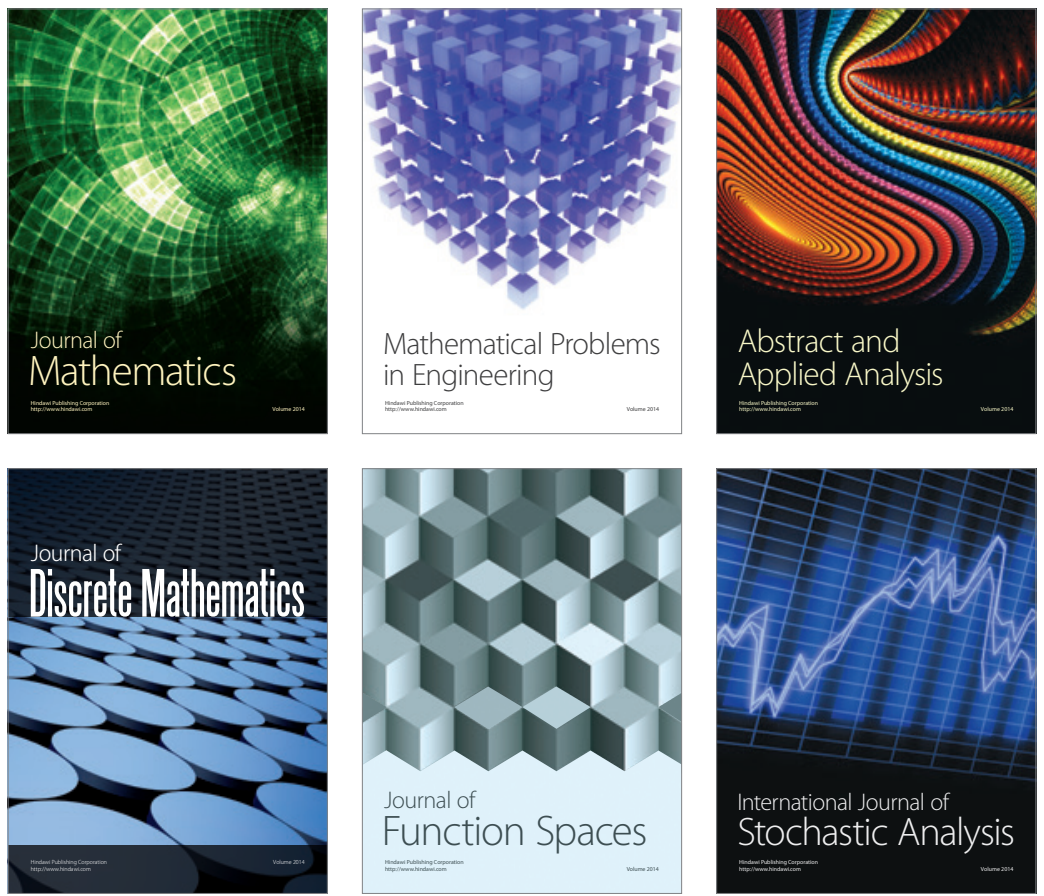

Journal of

Function Spaces

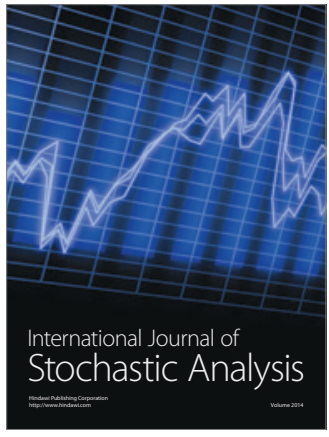

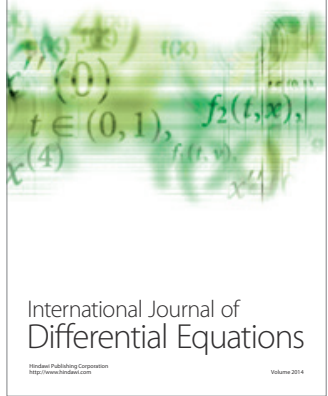
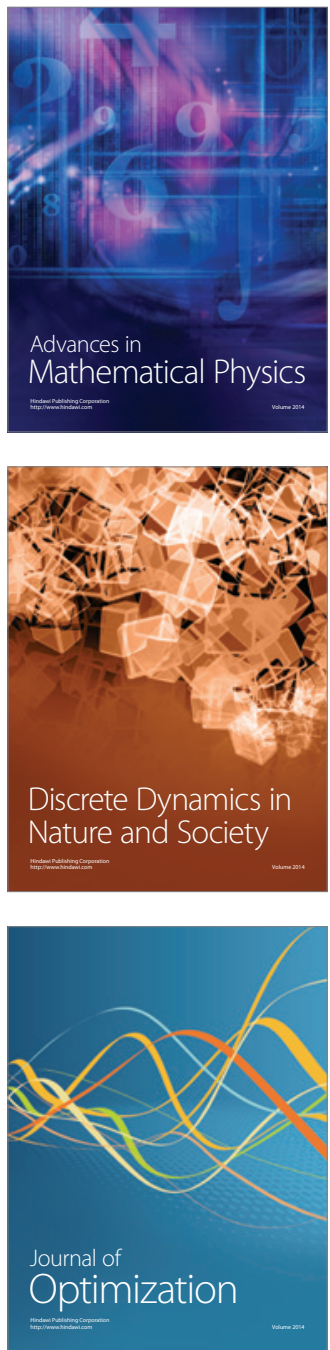